\title{
Characterization of Acylated Anthocyanins in Callus Induced From Storage Root of Purple-Fleshed Sweet Potato, Ipomoea batatas L
}

\author{
N. Terahara, ${ }^{1 *}$ I. Konczak, ${ }^{2}$ H. Ono, ${ }^{3}$ M. Yoshimoto, ${ }^{4}$ and O. Yamakawa ${ }^{5}$ \\ ${ }^{1}$ Department of Food Science for Health, Faculty of Health and Nutrition, Minami-Kyushu University, \\ 5-1-2 Kirishima, Miyazaki 880-0032, Japan \\ ${ }^{2}$ Food Science Australia, CRC for Bioproducts, 16 Julius Avenue, Riverside Corporate Park, \\ North Ryde, NSW 2113, Australia \\ ${ }^{3}$ National Food Research Institute, Tsukuba, Ibaraki 305-8642, Japan \\ ${ }^{4}$ National Agricultural Research Centre for Kyushu Okinawa Region, Miyakonojo, Miyazaki 885-0091, Japan \\ ${ }^{5}$ National Agricultural Research Centre for Kyushu Okinawa Region, Nishigoshi, Kikuchi, Kumamoto 861-1192, Japan
}

Received 4 June 2004; revised 8 June 2004; accepted 15 June 2004

\begin{abstract}
Four anthocyanins were isolated from a highly pigmented callus induced from the storage root of purple-fleshed sweet potato (Ipomoea batatas L) cultivar Ayamurasaki. The anthocyanins were respectively identified as cyanidin 3-O-(2-O-(6-O(E)-caffeoyl- $\beta$-D-glucopyranosyl)- $\beta$-D-glucopyranoside)-5-O- $\beta$-D-glucopyranoside, cyanidin 3-O-(2-O-(6-O- $(E)$ - $p$-coumaroyl- $\beta$ D-glucopyranosyl)-6-O-(E)-caffeoyl- $\beta$-D-glucopyranoside)-5-O- $\beta$-D-glucopyranoside, cyanidin 3-O-(2-O-(6-O-(E)- $p$-coumaroyl$\beta$-D-glucopyranosyl)-6-O-(E)-p-coumaroyl- $\beta$-D-glucopyranoside)-5-O- $\beta$-D-glucopyranoside, and peonidin 3-O-(2-O-(6-O- $(E)-$ $p$-coumaroyl- $\beta$-D-glucopyranosyl)-6- $O-(E)$ - $p$-coumaroyl- $\beta$-D-glucopyranoside)-5- $O$ - $\beta$-D-glucopyranoside by chemical and spectroscopic analyses. These anthocyanins were examined with respect to the stability in neutral aqueous solution as well as the radical scavenging activity against the 1,1-diphenyl-2-picrylhydrazyl (DPPH) radical. These acylated anthocyanins exhibited both higher stability and higher DPPH radical scavenging activity than corresponding nonacylated cyanidin and peonidin 3-O-sophoroside-5-Oglucosides.
\end{abstract}

\section{INTRODUCTION}

A purple-fleshed sweet potato, Ipomoea batatas L cultivar Ayamurasaki, accumulates high levels of anthocyanin pigments in the storage root [1]. The major anthocyanins are cyanidin and peonidin 3-Osophoroside-5-O-glucosides acylated with caffeic, ferulic, or $p$-hydroxybenzoic acids $[2,3,4]$. The sweet potato anthocyanins possess not only moderate stability in neutral aqueous solution [5] and high thermostability [6] but also various health benefits like antioxidative activity $[7,8,9]$, antimutagenicity $[10,11]$, and antidiabetic action $[12,13,14]$. Therefore, the Ayamurasaki pigment is utilized as a high-quality natural food colorant with potentially preventive function against life-style-related diseases.

Recently, from the storage root of the cultivar Ayamurasaki we have established a high-anthocyanin accumulating cell line. In the crude pigment extract, some cellline-specific anthocyanins have been detected which were different from those in the original storage root [15]. Previously, we have isolated and determined the molecular structures of the two anthocyanins, in which one was a known anthocyanin, cyanidin 3-sophoroside-5-glucoside, and the other was a new one, cyanidin 3-( $p$-coumaroyl) sophoroside-5-glucoside [5]. In sequence, we have isolated four more callus anthocyanins. The purpose of this study is to characterize the structures and the stability in a neutral aqueous solution and the antioxidative activity to utilize the pigment for food or other material.

\section{MATERIALS AND METHODS}

\section{Chemicals}

All the reagents and solvents employed were of analytical grade and used without further purification. 1,1diphenyl-2-picrylhydrazyl (DPPH, Wako Pure Chemicals, Japan), $\alpha$-tocopherol (Kishida Chemicals, Japan), and 2,6di-tert-butyl-4-methylphenol (BHT, Wako Pure Chemicals) were used for DPPH radical scavenging assay. Open column chromatographies were carried out on Amberlite XAD-2000 (Rohm and Haas, USA) and ODS (Silica Gel Chromatorex, Fuji Silysia Chemical Ltd, Japan) resins. 


\section{HPLC methods}

Solvent $\mathrm{A}\left(1.5 \% \mathrm{H}_{3} \mathrm{PO}_{4}\right.$ in $\left.\mathrm{H}_{2} \mathrm{O}\right)$ and solvent $\mathrm{B}(1.5 \%$ $\mathrm{H}_{3} \mathrm{PO}_{4}, 20 \% \mathrm{CH}_{3} \mathrm{COOH}, 25 \% \mathrm{MeCN}$ in $\mathrm{H}_{2} \mathrm{O}$ ) were used for analytical high-performance liquid chromatography (HPLC), and solvent $\mathrm{A}\left(15 \% \mathrm{CH}_{3} \mathrm{COOH}\right.$ in $\left.\mathrm{H}_{2} \mathrm{O}\right)$ and solvent $\mathrm{B}\left(15 \% \mathrm{CH}_{3} \mathrm{COOH}, 30 \% \mathrm{MeCN}\right.$ in $\left.\mathrm{H}_{2} \mathrm{O}\right)$ were used for preparative HPLC. Analytical HPLC was run on LC-10AD intelligent pump (Shimadzu, Japan) with a linear gradient elution of solvent $\mathrm{A}: \mathrm{B}=75: 25$ to 15 : 85 for 40 minutes on Luna column (4.6 id $\times$ $100 \mathrm{~mm}$, Phenomenex, USA) at $35^{\circ} \mathrm{C}$ with a flow rate of $1.0 \mathrm{~mL} / \mathrm{min}$ monitoring at $520 \mathrm{~nm}$ equipped with SPD10A diode array detector (Shimadzu). For purity check of anthocyanins, other analytical HPLC was performed on L-6200 intelligent pump system (Hitachi, Japan) with a linear gradient elution of solvent $\mathrm{A}: \mathrm{B}=75: 25$ to 45 : 55 for 60 minutes on Inertsil ODS-3 column (4.6 id $\times 250 \mathrm{~mm}$, GL Sciences, Japan) at $30^{\circ} \mathrm{C}$ with a flow rate of $1.0 \mathrm{~mL} / \mathrm{min}$ monitoring at 520,310 , or $280 \mathrm{~nm}$ equipped with MD-1510 multiwavelength detector (Jasco, Japan). Preparative HPLC was run on L-6200 intelligent pump system with isocratic elutions of solvent $\mathrm{A}: \mathrm{B}=80: 20$ for $1,75: 25$ for $2,60: 40$ for 3 , and $50: 50$ for 4 on Inertsil ODS column $(20 \mathrm{id} \times 250 \mathrm{~mm}$, GL Sciences $)$ at room temperature with a flow rate of $7.0 \mathrm{~mL} / \mathrm{min}$ monitoring at $310 \mathrm{~nm}$ with L-4200 UV-Vis detector and D-2000 integrator (Hitachi).

\section{Spectral analyses}

UV-Vis spectra were recorded on V-550 spectrophotometer (Jasco) in $0.01 \% \mathrm{HCl}-\mathrm{MeOH}$ for structural determination and for colorimetric measurements of antioxidative activity assay. The bathochromic shift test was carried out by the addition of $5 \% \mathrm{AlCl}_{3}-\mathrm{MeOH}$. Electrospray ionization time-of-flight mass spectrometry (ESI-TOFMS) spectra were recorded on Mariner Workstation system (Applied Biosystems, USA) in 1\% $\mathrm{CH}_{3} \mathrm{COOH}-50 \% \mathrm{MeCN}$ with a positive mode by an infusion-injection. High-resolutional electrospray ionization Fourier-transform ion-cyclotron resonance mass spectrometry (ESI/FT-ICRMS) spectra were recorded on APEX II 70e (Bruker Daltonics, Germany) in aqueous $\mathrm{MeOH}$ containing $\mathrm{CH}_{3} \mathrm{COOH}$ with a positive mode. ${ }^{1} \mathrm{H}$ $(800 \mathrm{MHz})$ and ${ }^{13} \mathrm{C}(200 \mathrm{MHz})$ NMR spectra were measured on Avance 800 spectrometer (Bruker BioSpin, Germany) in DMSO- $d_{6}:$ TFA- $d_{1}=9: 1$ with tetramethylsilane (TMS) as an internal standard at $30^{\circ} \mathrm{C}$.

\section{Plant materials}

A previously established high-anthocyanin-accumulating callus culture generated from the storage root of sweet potato cultivar Ayamurasaki has been used for this study [15]. Suspended cell cultures were initiated by transferring about $1.0 \mathrm{~g}$ (fresh weight) of callus to $50 \mathrm{~mL}$ of liquid medium in $250 \mathrm{~mL}$ Erlenmeyer flasks. Basal Murashige and Skoog (MS) medium [16] supplemented with $1.0 \mathrm{mg} / \mathrm{L} \mathrm{2,4-D}$, and $3 \%$ sucrose has been used as a maintenance medium (MM). Medium $\mathrm{pH}$ has been adjusted to 5.8 before autoclaving. Subcultures have been done in 7-day intervals. The cultures were incubated on a rotary shaker $(130 \mathrm{rpm})$ at $25^{\circ} \mathrm{C}$ in the dark. For the purpose of present analysis, suspension cultures have been produced under two different medium conditions: MM and a high-anthocyanin producing medium (APM).

The APM was a modified MS medium with $9.4 \mathrm{mM}$ $\mathrm{KNO}_{3}$, without $\mathrm{NH}_{4} \mathrm{NO}_{3}$, with $5 \%$ sucrose and nil growth regulators [17]. Medium $\mathrm{pH}$ has been adjusted to 5.8 before autoclaving and suspension cultures were incubated in $250 \mathrm{~mL}$ flasks. Five hundred mg of cell aggregates were placed in flasks containing $50 \mathrm{~mL}$ medium. The cultures were harvested after 7 days' growth on MM (200 g) and after 14 days on APM (199g).

\section{Extraction and isolation of anthocyanins}

The aggregates were removed from the culture media, rinsed with distilled water, separated from the liquid by vacuum filtration, and weighed. A total amount of $399 \mathrm{~g}$ of fresh tissue was steeped in $15 \% \mathrm{CH}_{3} \mathrm{COOH}(1 \mathrm{~L})$ for one day and filtered. This operation was repeated three more times $(1,1$, and $0.5 \mathrm{~L})$. The combined crude extract (3.5 L) contained nineteen or more anthocyanins [18]. The extract solution was applied on two XAD-2000 resin columns $(30 \mathrm{id} \times 385 \mathrm{~mm})$, the columns were washed with water $(3 \mathrm{~L})$, eluted stepwise with $10 \%, 20 \%, 30 \%$, $40 \%, 60 \%$, or $70 \% \mathrm{EtOH}$ all with $1 \% \mathrm{CH}_{3} \mathrm{COOH}(1 \mathrm{~L})$. Pigments containing 30\%, 40\%, 60\%, and 70\% EtOH fractions were combined and evaporated to dryness under reduced pressure. Subsequently, the residue was separated using ODS column (60 id $\times 320 \mathrm{~mm})$ with $10 \%$, 20\%, $30 \%, 40 \%, 60 \%$, or $70 \%$ EtOH all with $1 \% \mathrm{CH}_{3} \mathrm{COOH}$ ( $1 \mathrm{~L})$. HPLC analysis confirmed that 1 was contained in $20 \% \mathrm{EtOH}$ fraction, 2 and 3 in 60\% EtOH fraction, and 4 in $70 \% \mathrm{EtOH}$ fraction, and each fraction was evaporated to dryness under reduced pressure. Finally, 1, 2, 3, and 4 were isolated on purification of the individual fractions by preparative HPLC monitoring at $310 \mathrm{~nm}$. The elutions were evaporated to dryness, dissolved in minimum amount of TFA, precipitated with excess ether, and dried in a silica gel desiccator under reduced pressure.

\section{Chemical analysis}

Alkaline hydrolysis of isolated pigment was performed as follows. The pigment powder ( $3 \mathrm{mg}$ ) was dissolved in $2 \mathrm{~N} \mathrm{NaOH}$, left for 15 minutes with a sealed cap, and then acidified with $\mathrm{CH}_{3} \mathrm{COOH}$. The components in the reaction mixture were identified by analytical HPLC.

Cyanidin

3-O-(2-O-(6-O-(E)-caffeoyl- $\beta$-D-glucopyranosyl)- $\beta-D-$ glucopyranoside)-5-O- $\beta$-D-glucopyranoside(1)

UV-Vis $\lambda_{\max }(0.01 \% \mathrm{HCl}-\mathrm{MeOH}) \mathrm{nm}: 526$ (bathochromic shift with $\mathrm{AlCl}_{3}$ into $\left.39 \mathrm{~nm}\right), 331,281$, $\mathrm{E}_{440} / \mathrm{E}_{\mathrm{vis} \cdot \max }=\mathrm{E}_{440} / \mathrm{E}_{526}=0.14, \mathrm{E}_{\mathrm{acyl} \cdot \max } / \mathrm{E}_{\mathrm{vis} \cdot \max }=$ 
$\mathrm{E}_{331} / \mathrm{E}_{526}=0.49 ;$ ESI-TOFMS: $\mathrm{m} / \mathrm{z} 935\left(\mathrm{M}^{+}=\right.$ $\left.\mathrm{C}_{42} \mathrm{H}_{47} \mathrm{O}_{24}{ }^{+}\right), 287\left(\mathrm{Cy}^{+}=\mathrm{C}_{15} \mathrm{H}_{11} \mathrm{O}_{6}{ }^{+}\right)$; high-resolutional ESI/FT-ICRMS: m/z 935.24620 (calculated 935.24518 for $\left.\mathrm{M}^{+}=\mathrm{C}_{42} \mathrm{H}_{47} \mathrm{O}_{24}{ }^{+}\right) ;{ }^{13} \mathrm{C}$ and ${ }^{1} \mathrm{H}$ NMR data were listed in Table 1 .

$$
\begin{gathered}
\text { Cyanidin 3-O-(2-O-(6-O-(E)-p-coumaroyl- } \beta-D- \\
\text { glucopyranosyl)-6-O-(E)-caffeoyl- } \beta-D- \\
\text { glucopyranoside)-5-O- } \beta-D-\text { glucopyranoside (2) }
\end{gathered}
$$

UV-Vis $\lambda_{\max }(0.01 \% \mathrm{HCl}-\mathrm{MeOH}) \mathrm{nm}: 528$ (bathochromic shift with $\mathrm{AlCl}_{3}$ into $\left.44 \mathrm{~nm}\right), 320,297,283$, $\mathrm{E}_{440} / \mathrm{E}_{\mathrm{vis} \cdot \max }=\mathrm{E}_{440} / \mathrm{E}_{528}=0.14, \mathrm{E}_{\mathrm{acyl} \cdot \max } / \mathrm{E}_{\mathrm{vis} \cdot \max }=$ $\mathrm{E}_{330} / \mathrm{E}_{528}=1.02 ;$ ESI-TOFMS: $\mathrm{m} / \mathrm{z} 1081\left(\mathrm{M}^{+}=\right.$ $\left.\mathrm{C}_{51} \mathrm{H}_{53} \mathrm{O}_{26}{ }^{+}\right), 287\left(\mathrm{Cy}^{+}=\mathrm{C}_{15} \mathrm{H}_{11} \mathrm{O}_{6}{ }^{+}\right)$; high-resolutional ESI/FT-ICRMS: m/z 1081.28241 (calculated 1081.28196 for $\left.\mathrm{M}^{+}=\mathrm{C}_{51} \mathrm{H}_{53} \mathrm{O}_{26}{ }^{+}\right) ;{ }^{13} \mathrm{C}$ and ${ }^{1} \mathrm{H}$ NMR data were listed in Table 1 .

\section{Cyanidin 3-O-(2-O-(6-O-(E)-p-coumaroyl- $\beta-D$ - glucopyranosyl)-6-O-(E)-p-coumaroyl- $\beta-D$ - glucopyranoside)-5-O- $\beta$-D-glucopyranoside (3)}

UV-Vis $\lambda_{\max }(0.01 \% \mathrm{HCl}-\mathrm{MeOH}) \mathrm{nm}: 529$ (bathochromic shift with $\mathrm{AlCl}_{3}$ into $\left.48 \mathrm{~nm}\right), 315,299$, $\mathrm{E}_{440} / \mathrm{E}_{\mathrm{vis} \text { max }}=\mathrm{E}_{440} / \mathrm{E}_{529}=0.15, \mathrm{E}_{\mathrm{acyl} \max } / \mathrm{E}_{\mathrm{vis} \cdot \max }=$ $\mathrm{E}_{315} / \mathrm{E}_{529}=1.21 ;$ ESI-TOFMS: $\mathrm{m} / \mathrm{z} 1065\left(\mathrm{M}^{+}=\right.$ $\left.\mathrm{C}_{51} \mathrm{H}_{53} \mathrm{O}_{25}{ }^{+}\right), 287\left(\mathrm{Cy}^{+}=\mathrm{C}_{15} \mathrm{H}_{11} \mathrm{O}_{6}{ }^{+}\right)$; high-resolutional ESI/FT-ICRMS: m/z 1065.28613 (calculated 1065.28704 for $\left.\mathrm{M}^{+}=\mathrm{C}_{51} \mathrm{H}_{53} \mathrm{O}_{25}{ }^{+}\right) ;{ }^{13} \mathrm{C}$ and ${ }^{1} \mathrm{H}$ NMR data were listed in Table 1.

Peonidin 3-O-(2-O-(6-O-(E)-p-coumaroyl- $\beta$ $D$-glucopyranosyl)-6-O-(E)-p-coumaroyl- $\beta-D$ glucopyranoside)-5-O- $\beta$-D-glucopyranoside (4)

UV-Vis $\lambda_{\max }(0.01 \% \quad \mathrm{HCl}-\mathrm{MeOH}) \mathrm{nm}: 528$ (no bathochromic shift with $\left.\mathrm{AlCl}_{3}\right), 315,297, \mathrm{E}_{440} / \mathrm{E}_{\mathrm{vis} \max }=$ $\mathrm{E}_{440} / \mathrm{E}_{528}=0.13, \mathrm{E}_{\text {acyl.max }} / \mathrm{E}_{\text {vis.max }}=\mathrm{E}_{315} / \mathrm{E}_{528}=$ 1.25; ESI-TOFMS: $\mathrm{m} / \mathrm{z} 1079\left(\mathrm{M}^{+}=\mathrm{C}_{52} \mathrm{H}_{55} \mathrm{O}_{25}{ }^{+}\right), 301$ $\left(\mathrm{Pn}^{+}=\mathrm{C}_{16} \mathrm{H}_{16} \mathrm{O}_{6}{ }^{+}\right)$; high-resolutional ESI/FT-ICRMS: $\mathrm{m} / \mathrm{z} 1079.30256$ (calculated 1079.30269 for $\mathrm{M}^{+}=$ $\mathrm{C}_{52} \mathrm{H}_{55} \mathrm{O}_{25}{ }^{+}$); ${ }^{13} \mathrm{C}$ and ${ }^{1} \mathrm{H}$ NMR data were listed in Table 1.

\section{Stability test}

Stability of anthocyanins 1, 2, 3, and 4 in neutral aqueous solution was compared with that of nonacylated anthocyanins cyanidin 3-O-sophoroside-5-O-glucoside (Cy3S5G) and peonidin 3-O-sophoroside-5-O-glucoside (Pn3S5G) isolated from original storage root according to a previously reported method [19]. Each anthocyanin TFA salt was dissolved in McIlvaine ( $\mathrm{pH}$ 7.0, $0.1 \mathrm{M}$ citrate- $0.2 \mathrm{M}$ phosphate) buffer solution to make $50 \mu \mathrm{M}$ test solution, and the Vis spectra $(400-700 \mathrm{~nm})$ were measured automatically at appropriate time intervals. Based on the absorbance at $\lambda_{\text {vis.max }}$ of each spectrum, the residual color (\%) was calculated as percent of the initial absorbance $(=100 \%)$. The stability of the anthocyanin

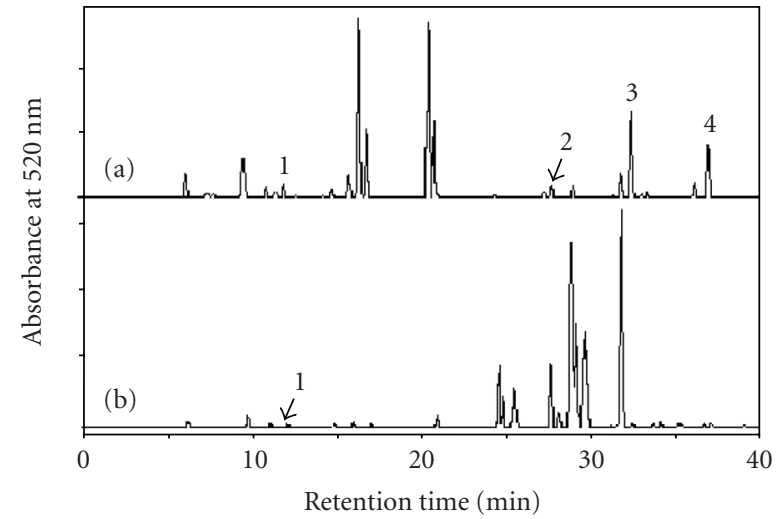

FIgURE 1. (a) HPLC chromatograms of the crude pigments of the purple sweet potato callus, and (b) storage root of sweet potato (Ipomoea batatas L), cv Ayamurasaki. Analytical HPLC was run with a linear gradient elution of solvent $\mathrm{A}: \mathrm{B}=85: 15$ to $65: 35$ for 100 minutes on Luna column at $35^{\circ} \mathrm{C}$ with a flow rate of $1.0 \mathrm{mLmin}^{-1}$ monitoring at $520 \mathrm{~nm}$.

could be evaluated on the basis of the half-life $\left(t_{1 / 2}\right)$, defined as the time required to reach $50 \%$ residual color.

\section{DPPH radical scavenging activity assay}

Radical scavenging activity of $1,2,3$, and 4 was tested according to the DPPH-colorimetric method developed by Yamaguchi et al [20] and compared with Cy3S5G, Pn3S5G, and $\alpha$-tocopherol as natural antioxidants and BHT as a synthetic antioxidant. Each sample was dissolved in EtOH to $500 \mu \mathrm{M}$ concentration. Sample solution $(25 \mu \mathrm{L})$ was added $375 \mu \mathrm{L}$ of EtOH, $350 \mu \mathrm{L}$ of Tris$\mathrm{HCl}$ buffer solution ( $\mathrm{pH} 7.4,0.1 \mathrm{M}$ ), and $250 \mu \mathrm{L}$ of $500 \mu \mathrm{M}$ $\mathrm{DPPH}-\mathrm{EtOH}$ solution (to obtain a final sample concentration was $12.5 \mu \mathrm{M}$ ), and immediately shaken and then kept standing for 20 minutes in the dark at room temperature. The absorbance of residual DPPH in sample solution was measured at $520 \mathrm{~nm}$. Initial and blank were measured without substrate and without DPPH, respectively. The DPPH radical scavenging activity (RS\%) was calculated as $R S \%=100\left(A_{i}-A_{s}+A_{b}\right) / A_{i}$, in which $A_{i}, A_{s}$, and $A_{b}$ were the absorbances at $520 \mathrm{~nm}$ of initial, sample, and blank solutions, respectively. The experiment was conducted with four replicates.

\section{RESULTS AND DISCUSSION}

The deep-purple callus induced from the storage root of purple-fleshed sweet potato cultivar Ayamurasaki was extracted with $15 \% \mathrm{CH}_{3} \mathrm{COOH}$. The crude extract contained 19 or more anthocyanins as detected by analytical HPLC (Figure 1(a)) [18]. The crude extract was successively purified by an absorbing resin and ODS column chromatographies and then preparative HPLC to afford four anthocyanins 1, 2, 3, and 4 (named as YGM-0d, $-3^{\prime}$, $-7 \mathrm{a}$, and $-7 \mathrm{e}$, resp) as red powders of TFA salts which were respectively obtained in amounts of 46, 28, 114, and $22 \mathrm{mg}$ (yield of $0.0115 \%, 0.007 \%, 0.0286 \%$, and $0.006 \%$, resp). 
TABLe 1. NMR chemical shifts purple-fleshed sweet potato callus anthocyanins $\mathbf{1}, \mathbf{2}, \mathbf{3}$, and 4 from tetramethylsilane in DMSO$d_{6} / \mathrm{TFA}_{-} d_{1}(9 / 1), 800 \mathrm{MHz}$.

\begin{tabular}{|c|c|c|c|c|c|c|c|c|}
\hline \multirow{2}{*}{ Position } & \multicolumn{2}{|r|}{1} & \multicolumn{2}{|r|}{2} & \multicolumn{2}{|r|}{3} & \multicolumn{2}{|r|}{4} \\
\hline & $\boldsymbol{\delta}_{C}$ & $\boldsymbol{\delta}_{H}$ & $\boldsymbol{\delta}_{C}$ & $\boldsymbol{\delta}_{H}$ & $\boldsymbol{\delta}_{C}$ & $\boldsymbol{\delta}_{H}$ & $\boldsymbol{\delta}_{C}$ & $\boldsymbol{\delta}_{H}$ \\
\hline \multicolumn{9}{|c|}{ Aglycon } \\
\hline 2 & 162.57 & - & 162.58 & - & 162.55 & - & 162.69 & - \\
\hline 3 & 144.59 & - & 144.36 & - & 144.28 & - & 144.39 & - \\
\hline 4 & 133.65 & $8.92 \mathrm{~s}$ & 133.35 & $8.27 \mathrm{~s}$ & 133.35 & $8.84 \mathrm{~s}$ & 135.62 & $8.95 \mathrm{~s}$ \\
\hline 5 & 155.31 & - & 155.41 & - & 155.37 & - & 155.63 & - \\
\hline 6 & 104.32 & $6.99 \mathrm{~d}(1.8)$ & 105.02 & $6.95 \mathrm{~d}(2.0)$ & 105.12 & $6.95 \mathrm{~d}(1.6)$ & 104.89 & $6.98 \mathrm{~d}(1.9)$ \\
\hline 7 & 167.80 & - & 167.92 & - & 167.91 & - & 168.24 & - \\
\hline 8 & 96.22 & $7.06 \mathrm{~d}(1.8)$ & 96.36 & $6.97 \mathrm{~d}(2.0)$ & 96.38 & $6.93 \mathrm{~d}(1.6)$ & 96.70 & $7.05 \mathrm{~d}(1.9)$ \\
\hline 9 & 111.74 & - & 111.88 & - & 111.87 & - & 112.00 & - \\
\hline 10 & 155.20 & - & 155.24 & - & 155.29 & - & 155.54 & - \\
\hline $1^{\prime}$ & 119.66 & - & 119.64 & - & 119.63 & - & 119.42 & - \\
\hline $2^{\prime}$ & 117.73 & $8.08 \mathrm{~d}(2.3)$ & 117.76 & $8.03 \mathrm{~d}(2.3)$ & 117.74 & $8.03 \mathrm{~d}(2.3)$ & 114.11 & $7.98 \mathrm{~d}(2.1)$ \\
\hline $3^{\prime}$ & 146.41 & - & 146.46 & - & 146.48 & - & 148.60 & - \\
\hline $4^{\prime}$ & 155.48 & - & 155.41 & - & 155.42 & - & 155.99 & - \\
\hline $5^{\prime}$ & 117.18 & $7.13 \mathrm{~d}(8.7)$ & 117.16 & $7.11 \mathrm{~d}(8.7)$ & 117.15 & $7.11 \mathrm{~d}(8.7)$ & 116.84 & $7.10 \mathrm{~d}(8.7)$ \\
\hline $6^{\prime}$ & 127.87 & $8.31 \mathrm{dd}(2.3,8.7)$ & 127.79 & $8.27 \mathrm{dd}(2.3,8.7)$ & 127.80 & $8.27 \mathrm{dd}(2.3,8.7)$ & 129.40 & $8.36 \mathrm{dd}(2.1,8.7)$ \\
\hline $\mathrm{OMe}$ & - & - & - & - & - & - & 56.19 & $3.90 \mathrm{~s}$ \\
\hline \multicolumn{9}{|c|}{ Glucose-a } \\
\hline 1 & 99.85 & $5.59 \mathrm{~d}(7.6)$ & 99.67 & $5.67 \mathrm{~d}(7.4)$ & 99.50 & $5.68 \mathrm{~d}(7.4)$ & 100.65 & $5.64 \mathrm{~d}(7.3)$ \\
\hline 2 & 81.54 & $4.02 \mathrm{t}(8.3)$ & 81.86 & $4.05 \mathrm{t}(8.1)$ & 81.84 & $4.06 \mathrm{t}(8.0)$ & 81.74 & $3.95 \mathrm{t}(7.6)$ \\
\hline 3 & 76.56 & $3.69 \mathrm{t}(8.9)$ & 76.06 & $3.76 \mathrm{t}(8.8)$ & 76.05 & $3.76 \mathrm{t}(8.8)$ & 75.95 & $3.75 \mathrm{t}(8.7)$ \\
\hline 4 & 69.43 & $3.39 \mathrm{t}(8.9)$ & 69.94 & $3.49 \mathrm{t}(9.3)$ & 69.98 & $3.48 \mathrm{t}(9.3)$ & 69.83 & $3.47 \mathrm{t}(9.2)$ \\
\hline 5 & 77.65 & $3.56 \mathrm{~m}$ & 74.27 & $3.93 \mathrm{~m}$ & 74.26 & $3.94 \mathrm{~m}$ & 74.44 & $3.87 \mathrm{~m}$ \\
\hline $6 a$ & 60.65 & $3.57 \mathrm{~m}$ & 63.20 & $4.32 \mathrm{dd}(6.8,12.2)$ & 63.24 & $4.31 \mathrm{dd}(7.1,12.1)$ & 63.16 & $4.27 \mathrm{dd}(6.8,12.6)$ \\
\hline $6 b$ & 60.65 & 3.74 brd (10.1) & 63.20 & 4.42 brd (10.0) & 63.24 & 4.42 brd (12.1) & 63.16 & 4.40 brd (11.3) \\
\hline \multicolumn{9}{|c|}{ Glucose-b } \\
\hline 1 & 104.44 & $4.84 \mathrm{~d}(7.8)$ & 104.71 & $4.81 \mathrm{~d}(7.8)$ & 104.69 & $4.82 \mathrm{~d}(7.8)$ & 104.47 & $4.78 \mathrm{~d}(7.8)$ \\
\hline 2 & 74.75 & $3.14 \mathrm{t}(8.4)$ & 74.85 & $3.15 \mathrm{t}(8.7)$ & 74.86 & $3.15 \mathrm{t}(8.4)$ & 74.65 & $3.19 \mathrm{t}(8.4)$ \\
\hline 3 & 76.26 & $3.27 \mathrm{t}(9.1)$ & 76.23 & $3.26 \mathrm{t}(8.7)$ & 76.24 & $3.27 \mathrm{t}(8.7)$ & 76.28 & $3.29 \mathrm{t}(8.9)$ \\
\hline 4 & 69.55 & $3.24 \mathrm{t}(8.8)$ & 69.61 & $3.25 \mathrm{t}(8.7)$ & 69.66 & $3.26 \mathrm{t}(8.8)$ & 69.77 & $3.26 \mathrm{t}(9.4)$ \\
\hline 5 & 74.18 & $3.14 \mathrm{~m}$ & 74.20 & $3.18 \mathrm{~m}$ & 74.21 & $3.19 \mathrm{~m}$ & 74.30 & $3.30 \mathrm{~m}$ \\
\hline $6 a$ & 62.58 & $3.99 \mathrm{dd}(6.2,12.5)$ & 62.66 & $3.99 \mathrm{dd}(4.3,11.8)$ & 62.67 & $4.00 \mathrm{dd}(4.3,11.7)$ & 63.23 & $4.03 \mathrm{dd}(5.4,11.6)$ \\
\hline $6 b$ & 62.58 & 3.92 brd (10.1) & 62.66 & 3.95 brd (10.0) & 62.67 & 3.96 brd (10.4) & 63.23 & 4.13 brd (10.1) \\
\hline \multicolumn{9}{|c|}{ Glucose-c } \\
\hline 1 & 101.59 & $5.12 \mathrm{~d}(7.7)$ & 102.24 & $5.07 \mathrm{~d}(7.7)$ & 102.35 & $5.05 \mathrm{~d}(7.7)$ & 102.01 & $5.10 \mathrm{~d}(7.7)$ \\
\hline 2 & 73.19 & $3.51 \mathrm{t}(8.5)$ & 73.39 & $3.56 \mathrm{t}(8.5)$ & 73.41 & $3.56 \mathrm{t}(8.9)$ & 73.35 & $3.56 \mathrm{t}(8.5)$ \\
\hline 3 & 76.02 & $3.41 \mathrm{t}(8.9)$ & 76.29 & $3.41 \mathrm{t}(9.0)$ & 76.30 & $3.41 \mathrm{t}(9.0)$ & 76.37 & $3.42 \mathrm{t}(9.0)$ \\
\hline 4 & 69.70 & $3.31 \mathrm{t}(9.2)$ & 69.88 & $3.29 \mathrm{t}(9.2)$ & 69.94 & $3.30 \mathrm{t}(9.3)$ & 69.83 & $3.31 \mathrm{t}(9.4)$ \\
\hline 5 & 77.65 & $3.49 \mathrm{~m}$ & 77.86 & $3.50 \mathrm{~m}$ & 77.85 & $3.48 \mathrm{~m}$ & 77.82 & $3.50 \mathrm{~m}$ \\
\hline $6 a$ & 60.74 & $3.58 \mathrm{dd}(5.7,12.3)$ & 61.08 & $3.59 \mathrm{dd}(6.2,12.3)$ & 61.04 & $3.58 \mathrm{dd}(5.8,12.0)$ & 60.93 & $3.59 \mathrm{dd}(5.7,12.2)$ \\
\hline $6 \mathrm{~b}$ & 60.74 & 3.78 brd (11.8) & 61.08 & 3.83 brd (10.1) & 61.04 & $3.81 \mathrm{dd}(1.9,11.9)$ & 60.93 & $3.80 \mathrm{dd}(2.0,10.0)$ \\
\hline
\end{tabular}


Table 1. Continued.

\begin{tabular}{ccccccccc}
\hline $\mathrm{G}_{\mathrm{a}}$-Acid I & & & & & & & \\
1 & - & - & 125.74 & - & 125.20 & - & 125.19 & - \\
2 & - & - & 115.40 & $6.94 \mathrm{~d}(2.0)$ & 130.35 & $7.33 \mathrm{~d}(8.6)$ & 130.30 & $7.28 \mathrm{~d}(8.7)$ \\
3 & - & - & 145.70 & - & 115.98 & $6.78 \mathrm{~d}(8.7)$ & 115.95 & $6.76 \mathrm{~d}(8.7)$ \\
4 & - & - & 148.53 & - & 160.08 & - & 160.05 & - \\
5 & - & - & 115.88 & $6.77 \mathrm{~d}(8.7)$ & 115.98 & $6.78 \mathrm{~d}(8.7)$ & 115.95 & $6.76 \mathrm{~d}(8.7)$ \\
6 & - & - & 121.61 & $6.86 \mathrm{dd}(2.0,8.7)$ & 130.35 & $7.33 \mathrm{~d}(8.6)$ & 130.30 & $7.28 \mathrm{~d}(8.7)$ \\
$\alpha$ & - & - & 113.98 & $6.02 \mathrm{~d}(15.8)$ & 113.96 & $6.026 \mathrm{~d}(15.9)$ & 113.79 & $5.96 \mathrm{~d}(15.9)$ \\
$\beta$ & - & - & 145.53 & $7.24 \mathrm{~d}(15.8)$ & 144.82 & $7.24 \mathrm{~d}(15.8)$ & 144.76 & $7.23 \mathrm{~d}(15.8)$ \\
Carbonyl & - & - & 166.77 & - & 166.78 & - & 166.68 & - \\
\hline $\mathrm{G}_{\mathrm{b}}$-Acid II & & & & & & & & \\
1 & 125.72 & - & 125.19 & - & 125.17 & - & 125.12 & - \\
2 & 115.15 & $6.99 \mathrm{~d}(2.0)$ & 130.36 & $7.33 \mathrm{~d}(8.6)$ & 130.58 & $7.38 \mathrm{~d}(8.6)$ & 130.52 & $7.38 \mathrm{~d}(8.7)$ \\
3 & 145.25 & - & 116.03 & $6.78 \mathrm{~d}(8.7)$ & 116.03 & $6.79 \mathrm{~d}(8.7)$ & 115.97 & $6.80 \mathrm{~d}(8.7)$ \\
4 & 148.48 & - & 160.08 & - & 160.08 & - & 160.08 & - \\
5 & 116.01 & $6.77 \mathrm{~d}(8.3)$ & 116.03 & $6.78 \mathrm{~d}(8.7)$ & 116.03 & $6.79 \mathrm{~d}(8.7)$ & 115.97 & $6.80 \mathrm{~d}(8.7)$ \\
6 & 121.39 & $6.83 \mathrm{dd}(2.0,8.3)$ & 130.36 & $7.33 \mathrm{~d}(8.6)$ & 130.58 & $7.38 \mathrm{~d}(8.6)$ & 130.52 & $7.38 \mathrm{~d}(8.7)$ \\
$\alpha$ & 113.94 & $6.00 \mathrm{~d}(15.8)$ & 113.86 & $6.17 \mathrm{~d}(15.8)$ & 113.86 & $6.23 \mathrm{~d}(15.9)$ & 113.79 & $6.18 \mathrm{~d}(15.9)$ \\
$\beta$ & 145.65 & $7.22 \mathrm{~d}(15.8)$ & 144.83 & $7.32 \mathrm{~d}(15.8)$ & 145.30 & $7.37 \mathrm{~d}(15.8)$ & 145.24 & $7.36 \mathrm{~d}(15.7)$ \\
Carbonyl & 166.46 & - & 166.48 & - & 166.49 & - & 166.47 & - \\
\hline
\end{tabular}

*Values in parentheses indicate coupling constants $(\mathrm{J}$ in $\mathrm{Hz})$.

**Abbreviations: $\mathrm{s}=$ singlet, $\mathrm{d}=$ doublet, $\mathrm{t}=$ triplet, $\mathrm{m}=$ multiplet, $\mathrm{dd}=$ double doublet, and $\mathrm{brd}=$ broad doublet.

On alkaline hydrolysis, 1, 2, and 3 gave cyanidin 3-O-sophoroside-5-O-glucoside (Cy3S5G) [19], while only 4 gave peonidin 3 -O-sophoroside-5-O-glucoside (Pn3S5G). Moreover, in the hydrolysates of 1, 2, 3, and 4 , caffeic acid from 1, caffeic acid and $p$-coumaric acid from 2, $p$-coumaric acid from 3 , and $p$-coumaric acid from 4 were detected as acylating acid(s) by HPLC cochromatographic analysis with authentic cinnamic acids. In the UV-Vis spectra, the absorptions around 331 and $320 \mathrm{~nm}$ of 1 and $2,315 \mathrm{~nm}$ of 3 and 4 also respectively supported the presence of caffeoyl and $p$-coumaroyl residues in their molecules. Numbers of the aromatic acids were respectively estimated one for $\mathbf{1}$, and two for 2,3 , and 4, on the basis of $\mathrm{E}_{\text {acyl.max }} / \mathrm{E}_{\mathrm{vis.max}}$ (absorbance at $\lambda_{\text {acyl.max }} /$ absorbance at $\lambda_{\text {vis.max }}$ ) values of 0.49 for 1 , and $1.02-1.25$ for 2,3 , and 4 , respectively.

Since absorption maxima $\left(\lambda_{\text {vis.max }}\right)$ of 1,2 , and 3 showed bathochromic shift by addition of $\mathrm{AlCl}_{3}$, but not in $4,1,2$, and 3 could be elucidated as cyanidin-(Cy)based anthocyanins and $\mathbf{4}$ as a peonidin-(Pn)-based one. ESI-TOFMS measurement of $\mathbf{1}, \mathbf{2}, \mathbf{3}$, and $\mathbf{4}$ showed molecular ion peaks at $\mathrm{m} / \mathrm{z} 935,1081,1065$, and 1079 corresponding to $\mathrm{C}_{42} \mathrm{H}_{47} \mathrm{O}_{24}{ }^{+}, \mathrm{C}_{51} \mathrm{H}_{53} \mathrm{O}_{26}{ }^{+}, \mathrm{C}_{51} \mathrm{H}_{53} \mathrm{O}_{25}{ }^{+}$, and $\mathrm{C}_{52} \mathrm{H}_{55} \mathrm{O}_{25}{ }^{+}$, respectively, and also showed fragment ion peaks of $\mathrm{Cy}^{+}$at $\mathrm{m} / \mathrm{z} 287$ in 1, 2, and 3 and of $\mathrm{Pn}^{+}$at $\mathrm{m} / \mathrm{z}$ 301 in $\mathbf{4}$. The molecular formulas of $\mathbf{1}, \mathbf{2}, \mathbf{3}$, and $\mathbf{4}$ were confirmed by high-resolutional ESI/FT-ICRMS at m/z $935.24620,1081.28241,1065.28613$, and 1079.30256, respectively. All these findings indicated $\mathbf{1}$ as mono-caffeoyl
Cy3S5G, 2 as caffeoyl-p-coumaroyl Cy3S5G, 3 as di- $p$ coumaroyl Cy3S5G, and 4 as di- $p$-coumaroyl Pn3S5G.

The complete structures of $\mathbf{1}, 2,3$, and $\mathbf{4}$ were established by ${ }^{13} \mathrm{C}$ and ${ }^{1} \mathrm{H}$ NMR analyses containing $2 \mathrm{D}$ pulse experiments such as a homonuclear double quantum filtered correlation spectroscopy (DQF-COSY), a total correlation spectroscopy (TOCSY), a heteronuclear singlequantum correlation (HSQC), a nuclear Overhauser and exchange spectroscopy (NOESY), and a heteronuclear multiple bond correlation spectroscopy (HMBC) technique. Assignment of ${ }^{13} \mathrm{C}$ - and ${ }^{1} \mathrm{H}$-signals was summarized in Table $1 .{ }^{1} \mathrm{H}$-signals in low magnetic field $\left(\delta_{\mathrm{H}} 6-\right.$ $9 \mathrm{ppm}$ ) shows characteristic aglycons and cinnamic acids. Anthocyanins 1, 2, and $\mathbf{3}$ and $\mathbf{4}$ have $\mathrm{Cy}$ and Pn moieties, respectively, due to the corresponding signals of benzopyrylium nucleus and 1,3,4-trisubstituted aromatic B-ring. Only 4 has an additional methoxyl signal at high magnetic field $\left(\delta_{\mathrm{H}} 3.90 \mathrm{ppm}, \delta_{\mathrm{C}} 56.19 \mathrm{ppm}\right)$. The presence of trans $(E)$-caffeoyl residue in $\mathbf{1}$ and $\mathbf{2}$ spectra and $(E)$ $p$-coumaroyl residue in 2,3 , and 4 spectra is confirmed with the 1,3,4-trisubstituted and 1, 4-disubstituted benzenes, respectively having the $(E)$-olefinic proton signals with large coupling constant (about $J_{\alpha, \beta}=16 \mathrm{~Hz}$ ). In high magnetic field $\left(\delta_{\mathrm{H}} 3-6 \mathrm{ppm}\right)$, the spectra also show all sugars of $1,2,3$, and 4 to be $\beta$-D-glucopyranosyl configuration because of the resonances at lower magnetic field $\left(\delta_{\mathrm{H}} 4.78-5.68 \mathrm{ppm}\right)$ of all anomeric protons and the large $J$ values $(J=7.2-9.4 \mathrm{~Hz})$ of the anomeric protons and the ring protons. As shown in Table 1, 


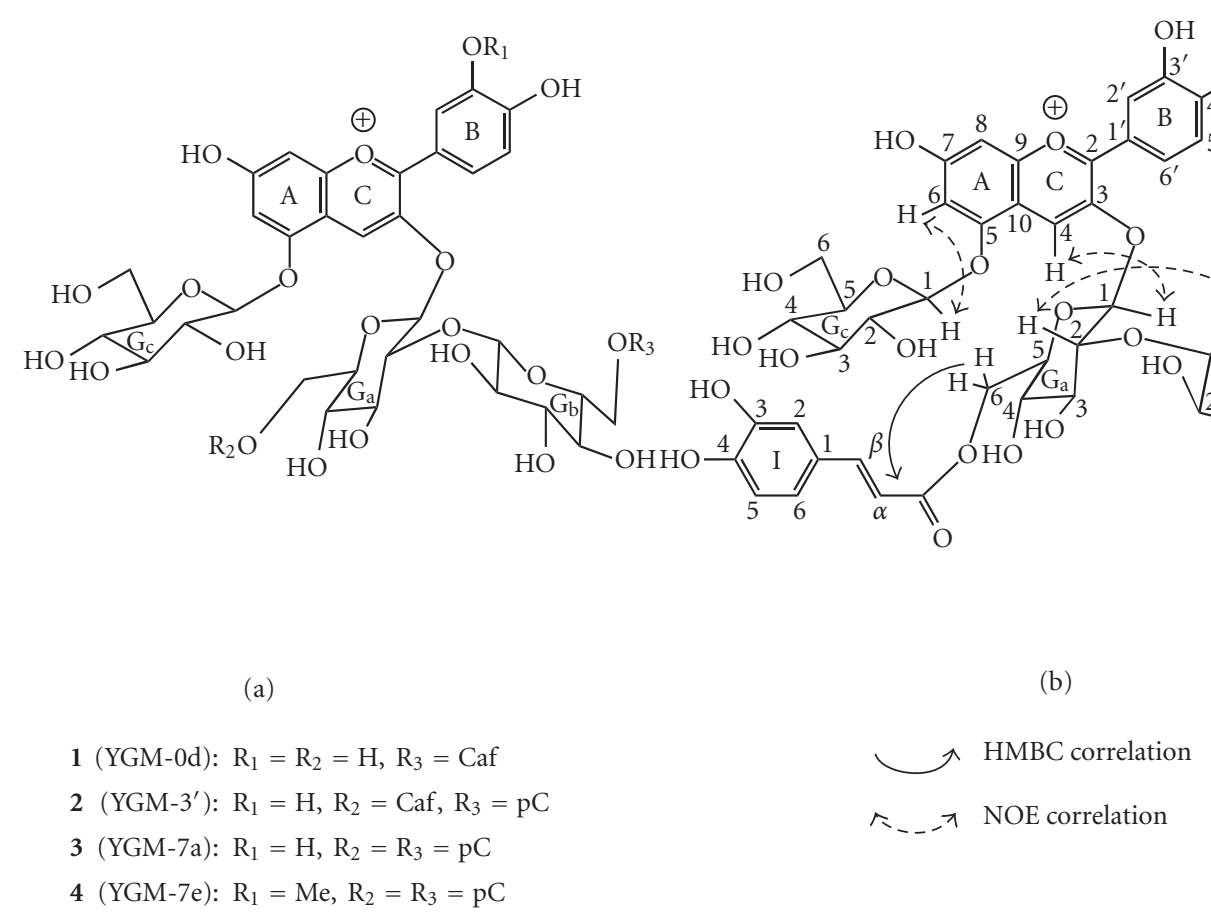

FIgURE 2. Purple sweet potato callus anthocyanins. (a) Structures of $1,2,3$, and 4 ; Caf $=$ caffeic acid and $p C=p$-coumaric acid, (b) typical NOE and HMBC correlations of caffeoyl- $p$-coumaroylated anthocyanin 2.

$\mathrm{G}_{\mathrm{a}}-2 \mathrm{H}($ at $\delta \mathrm{H} 3.95-4.06 \mathrm{ppm})$ and $\mathrm{G}_{\mathrm{a}}-2 \mathrm{C}(\delta \mathrm{C} 81.54-$ $81.86 \mathrm{ppm}$ ) clearly shift to downfield more than $\mathrm{G}_{\mathrm{b}}-2 \mathrm{H}$ (at $\delta \mathrm{H} 3.14-3.19 \mathrm{ppm})$ and $\mathrm{G}_{\mathrm{b}}-2 \mathrm{C}(\delta \mathrm{C} 74.65-74.86$ ppm) or $\mathrm{G}_{\mathrm{c}}-2 \mathrm{H}($ at $\delta \mathrm{H} 3.51-3.56 \mathrm{ppm})$ and $\mathrm{G}_{\mathrm{c}}-2 \mathrm{C}(\delta$ C 73.19-73.39 ppm). On the basis of this data, we concluded that glucose $b\left(G_{b}\right)$ links to glucose $a\left(G_{a}\right)-20 H$. On the other hand, NOESY and HMBC spectra gave more direct and certain data on the presence of $\beta-D-G_{b}(1 \rightarrow 2)$ $\mathrm{G}_{\mathrm{a}}$ bond of the sophorosyl residue.

The connecting relations among an aglycon, three sugars, and acyl groups in 1, 2, 3, and 4 were confirmed by NOESY and HMBC measurements, for example, anthocyanin 2 shown in Figure 2(b). In the NOESY spectra of $1,2,3$, and 4 , three intensive NOE signals between aglycon- $4 \mathrm{H}$ and $\mathrm{G}_{\mathrm{a}}-1 \mathrm{H}$ (aglycon- $4 \mathrm{H} / \mathrm{G}_{\mathrm{a}}-1 \mathrm{H}$ ), $\mathrm{G}_{\mathrm{a}}-2 \mathrm{H} / \mathrm{G}_{\mathrm{b}}-1 \mathrm{H}$, and aglycon- $6 \mathrm{H} / \mathrm{G}_{\mathrm{c}}-1 \mathrm{H}$ indicated that $\mathrm{G}_{\mathrm{a}}$, $\mathrm{G}_{\mathrm{b}}$, and $\mathrm{G}_{\mathrm{c}}$ connected at aglycon-3-OH, at $\mathrm{G}_{\mathrm{a}}-2 \mathrm{OH}$, and at aglycon-5OH through glycosyl bond, respectively. In the HMBC spectra of $1,2,3$, and 4 , the clear ${ }^{1} \mathrm{H}-{ }^{13} \mathrm{C}$ cross peaks between $\mathrm{G}_{\mathrm{a}}-1 \mathrm{H}$ and aglycon-3-carbon signals $\left(\mathrm{G}_{\mathrm{a}}-1 \mathrm{H} /\right.$ aglycon-3C), $\mathrm{G}_{\mathrm{b}}-1 \mathrm{H} / \mathrm{G}_{\mathrm{a}}-2 \mathrm{C}, \mathrm{G}_{\mathrm{a}}-2 \mathrm{H} / \mathrm{G}_{\mathrm{b}}-1 \mathrm{C}$, and $\mathrm{G}_{\mathrm{c}}-1 \mathrm{H} /$ aglycon-5C verified the connections of $\mathrm{G}_{\mathrm{a}}$ /aglycon-3OH, $\mathrm{G}_{\mathrm{b}} / \mathrm{G}_{\mathrm{a}}-2 \mathrm{OH}$, and $\mathrm{G}_{\mathrm{c}}$ /aglycon-5OH, respectively. Moreover, the distinct correlation peaks between $\mathrm{G}_{\mathrm{b}}-6 \mathrm{H}$ and acyl-carbonyl carbon signals (about $\delta_{\mathrm{C}} \quad 166.5 \mathrm{ppm}$ ) provided decisive proof that acylating acids were linked at $\mathrm{G}_{\mathrm{b}}-6 \mathrm{OH}$. In 2,3 , and 4 , the cross peak between $\mathrm{G}_{\mathrm{c}}-6 \mathrm{H}$ and acyl-carbonyl carbon signals (about $\delta_{\mathrm{C}} 166.8 \mathrm{ppm}$ ) also showed directly links aromatic acids and $\mathrm{G}_{\mathrm{c}}-6 \mathrm{OH}$ [3]. In conclusion, 1, 2, 3, and 4 were unambiguously determined as cyanidin 3-O-(2-O-(6-O(E)-caffeoyl- $\beta$-D-glucopyranosyl)- $\beta$-D-glucopyranoside) -5-O- $\beta$-D-glucopyranoside, cyanidin 3-O-(2-O-(6-O- $(E)$ - $p$-coumaroyl- $\beta$-D-glucopyranosyl)-6-O- $(E)$-caffeoyl- $\beta$ D-glucopyranoside)-5-O- $\beta$-D-glucopyranoside, cyanidin 3-O-(2-O-(6-O-(E)-p-coumaroyl- $\beta$-D-glucopyranosyl)6-O-(E)- $p$-coumaroyl- $\beta$-D-glucopyranoside)-5-O- $\beta$-Dglucopyranoside, and peonidin 3-O-(2-O-(6-O- $(E)-p-$ coumaroyl- $\beta$-D-glucopyranosyl)-6-O- $(E)-p$-coumaroyl$\beta$-D-glucopyranoside)-5-O- $\beta$-D-glucopyranoside, respectively, by chemical and spectroscopic analyses (Figure 2(a)). Only anthocyanin 4 is a new compound, while $\mathbf{1}, \mathbf{2}$, and 3 are known ones. Anthocyanins $\mathbf{1}$ and $\mathbf{2}$ have already been identified in Ipomoea cairica flowers [21], but the exact binding sites of the acyl residues were not established. Similarly, anthocyanins $\mathbf{2}$ and $\mathbf{3}$ have been identified in the pigment of Ipomoea asarifolia flower [22], and in the pigment of Ajuga reptans flower and the corresponding cell cultures [23], respectively. Pigment $\mathbf{1}$ is also found in original purple-fleshed sweet potato storage root (Figure 1(b)) or sweet potato leaf pigment as a minor component, while $p$-coumaroylated 2,3 , and 4 are confirmed to be cell-line-specific pigments.

The stability of $1,2,3$, and 4 was compared with nonacylated Cy3S5G and Pn3S5G in neutral aqueous solution at room temperature. The stability was evaluated on the basis of the half-life $\left(t_{1 / 2}\right)$ that was defined as the time required to reach $50 \%$ residual color. As shown in 


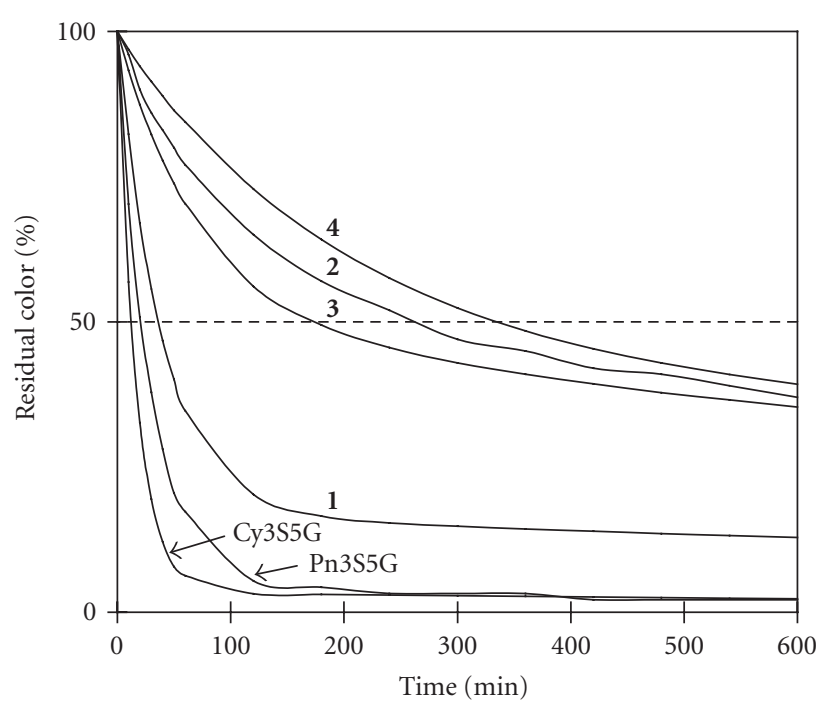

Figure 3. Stability of purple sweet potato callus anthocyanins $\mathbf{1}$, 2,3 , and 4 in neutral aqueous solution at room temperature.

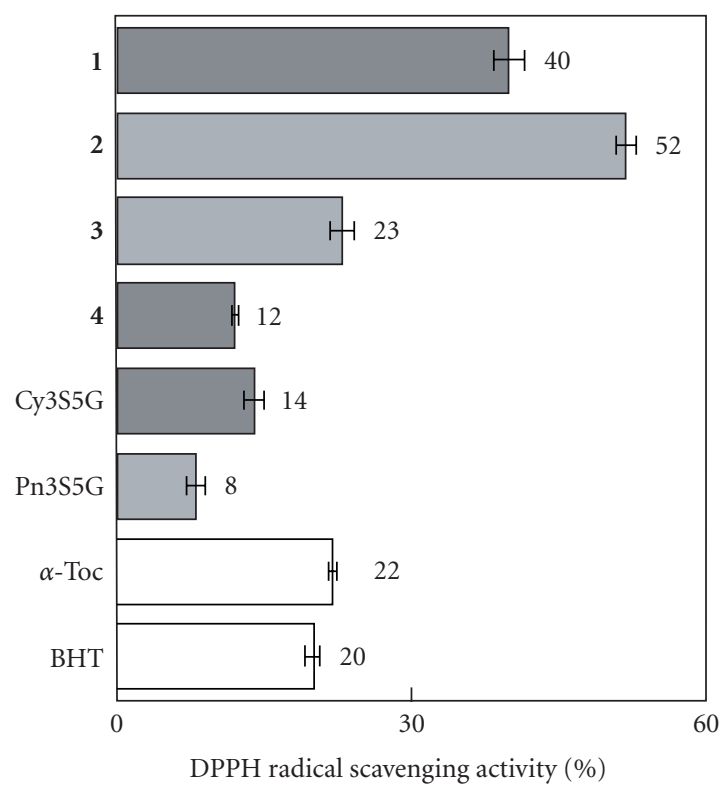

FIGURE 4. DPPH radical scavenging activity (\%) of purple sweet potato callus anthocyanins $1,2,3$, and $4(\mathrm{pH} 7.4$ at room temperature). $\alpha$-Toc $=\alpha$-tocopherol and BHT $=2,6$-di-tert-butyl-4methylphenol. Each value is the mean (RS\%) \pm standard deviation $(\mathrm{n}=4)$.

Figure 3, diacylated anthocyanins $\left(t_{1 / 2}\right.$ of 2: 266 minutes, 3: 180 minutes, and 4: 337 minutes) were more stable than monoacylated $\mathbf{1}\left(\mathrm{t}_{1 / 2}=37\right.$ minutes $)$, and nonacylated Cy3S5G ( $\mathrm{t}_{1 / 2}=15$ minutes $)$ and Pn3S5G $\left(\mathrm{t}_{1 / 2}=19\right.$ minutes). Effect of the structures on the stabilities of the anthocyanins suggested that the two aromatic acids protect the aglycon nucleus by a sandwich-type hydrophobic stacking mechanism and inhibit the attack of a water molecule that leads to a loss of color $[24,25]$.
These anthocyanins were evaluated for the antioxidant activity as scavenging ability against DPPH radical. As shown in Figure 4, activity of identically substituted but Cy-based 3 exhibited higher scavenging activity than Pn-based 4. The caffeoyl- $p$-coumaroylated 2 and monocaffeoylated 1 showed higher activity than di- $p$ coumaroylated 3 . Therefore, anthocyanins with catechol group(s) in the aglycon and/or the acyl group(s) were thought to be very effective in radical scavenging. Moreover, since hydroxycinnamic acid acylation enhanced the activity (1, 2, $3>$ Cy3S5G, and $4>$ Pn3S5G), the acylation might intramolecularly and synergistically achieve the radical scavenging activity of aglycon in addition to their own activity [26].

The cell line generated from Ayamurasaki storage root accumulates high levels of anthocyanin pigments. Depending on medium conditions under which the tissue is produced, the total amount of anthocyanins is equal to that of the original storage root on an MM [15] or 2.5-fold higher on a high-anthocyanin producing medium [27].

Thus the callus pigment has a potential to be utilized as a high-quality natural food colorant/natural food ingredient with protective action against oxidative damage [18].

\section{REFERENCES}

[1] Yoshinaga M. New cultivar "Ayamurasaki" for colorant production. Sweetpotato ResearchFront (KNAES). 1995;2.

[2] Odake K, Terahara N, Saito N, Toki K, Honda T. Chemical structures of two anthocyanins from purple sweet potato, Ipomoea batatas. Phytochemistry. 1992;31:2127-2130.

[3] Goda Y, Shimizu T, Kato Y, et al. Two acylated anthocyanins from purple sweet potato. Phytochemistry. 1997;44(1):183-186.

[4] Terahara N, Kato Y, Nakamura M, Maitani T, Yamaguchi M, Goda Y. Six diacylated anthocyanins from storage roots of purple sweet potato, Ipomoea batatas. Biosci Biotechnol Biochem. 1999;63(8):14201424.

[5] Terahara N, Konczak-Islam I, Nakatani M, Yamakawa O, Goda Y, Honda T. Anthocyanins in callus induced from purple storage root of Ipomoea batatas L. Phytochemistry. 2000;54(8):919-922.

[6] Odake K. Characteristics of food color pigments derived from Ayamurasaki. In: LaBonte DR, Yamashita M, Mochida M, eds. Proceedings of International Workshop on Sweet potato Production System Toward the 21 Century. Japan: KNAES; 1997:303-309.

[7] Furuta S, Suda I, Nishiba Y, Yamakawa O. High tertbutylperoxyl radical scavenging activities of sweetpotato cultivars with purple flesh. Food Science and Technology International. 1998;4:33-35.

[8] Terahara N. Structure and antioxidative activity of the stable acylated anthocyanins. In: Proc 2 nd 
International Conference on Food Factors-Chemistry and Health Promotion; Kyoto, Japan 1999. Supported by The Commemorative Association for The Japan World Exposition (1970).

[9] Oki T, Masuda M, Furuta S, Nishiba Y, Terahara N, Suda I. Involvement of anthocyanins and other phenolic compounds in radical-scavenging activity of purple-fleshed sweet potato cultivars. J Food Sci. 2002;67(5):1752-1756.

[10] Yoshimoto M, Okuno S, Yoshinaga M, Yamakawa O, Yamaguchi M, Yamada J. Antimutagenicity of sweet potato (Ipomoea batatas) roots. Biosci Biotechnol Biochem. 1999;63(3):537-541.

[11] Yoshimoto M, Okuno S, Yamaguchi M, Yamakawa O. Antimutagenicity of deacylated anthocyanins in purple-fleshed sweet potato. Biosci Biotechnol Biochem. 2001;65(7):1652-1655.

[12] Matsui T, Ueda T, Oki T, Sugita K, Terahara N, Matsumoto K. Alpha-glucosidase inhibitory action of natural acylated anthocyanins. 1. Survey of natural pigments with potent inhibitory activity. J Agric Food Chem. 2001;49(4):1948-1951.

[13] Matsui T, Ueda T, Oki T, Sugita K, Terahara N, Matsumoto K. Alpha-glucosidase inhibitory action of natural acylated anthocyanins. 2. Alpha-glucosidase inhibition by isolated acylated anthocyanins. J Agric Food Chem. 2001;49(4):1952-1956.

[14] Matsui T, Ebuchi S, Kobayashi M, et al. Anti-hyperglycemic effect of diacylated anthocyanin derived from Ipomoea batatas cultivar Ayamurasaki can be achieved through the Alpha-glucosidase inhibitory action. J Agric Food Chem. 2002;50(25):7244-7248.

[15] Konczak-Islam I, Yoshinaga M, Nakatani M, Terahara N, Yamakawa O. Establishment and characteristics of an anthocyanin-producing cell line from sweet potato storage root. Plant Cell Rep. 2000;19(5):472-477.

[16] Murashige T, Skoog F. A revised medium for rapid growth and bioassays with tobacco tissue cultures. Physiol Plant. 1962;15:473-497.

[17] Konczak-Islam I, Nakatani M, Yoshinaga M, Yamakawa O. Effect of ammonium ion and temperature on anthocyanin composition in sweet potato cell suspension culture. Plant Biotechnol. 2001;18:109-117.

[18] Konczak-Islam I, Yoshimoto M, Hou DX, Terahara N, Yamakawa O. Potential chemopreventive properties of anthocyanin-rich aqueous extracts from in vitro produced tissue of sweet potato (Ipomoea batatas L.). J Agric Food Chem. 2003;51(20):59165922.

[19] Terahara N, Callebaut A, Ohba R, Nagata T, Ohnishi-Kameyama M, Suzuki M. Triacylated anthocyanins from Ajuga reptans flowers and cell cultures. Phytochemistry. 1996;42(1):199-203.

[20] Yamaguchi T, Takamura H, Matoba T, Terao J. HPLC method for evaluation of the free radical-scavenging activity of foods by using 1,1-diphenyl-2- picrylhy- drazyl. Biosci Biotechnol Biochem. 1998;62(6):12011204.

[21] Pomilio AB, Sproviero JF. Acylated anthocyanins from Ipomoea cairica. Phytochemistry. 1972;11: 1125-1128.

[22] Pale E, Nacro M, Vanhaelen M, Vanhaelen-Fastre R, Ottinger R. Acylated anthocyanins from the flowers of Ipomoea asarifolia. Phytochemistry. 1998;48(8): 1433-1437.

[23] Terahara N, Callebaut A, Ohba R, Nagata T, Ohnishi-Kameyama M, Suzuki M. Acylated anthocyanidin 3-sophoroside-5-glucosides from Ajuga reptans flowers and the corresponding cell cultures. Phytochemistry. 2001;58(3):493-500.

[24] Brouillard, R. The in vivo expression of anthocyanin colour in plants. Phytochemistry. 1983;22:13111323.

[25] Goto T, Kondo T. Structure and molecular stacking of anthocyanins - flower color variation. Angew Chem Int Ed Engl. 1991;30(1):17-33.

[26] Tamura H, Yamagami A. Antioxidative activity of monoacylated anthocyanins isolated from Muscat Bailey A grape. J Agric Food Chem. 1994;42(8):16121615.

[27] Konczak-Islam I, Okuno S, Yoshimoto M, Yamakawa O. Composition of phenolics and anthocyanins in a sweet potato cell suspension culture. Biochem Eng J. 2003;14(3):155-161.

* Corresponding author.

E-mail: terahara@nankyudai.ac.jp

Fax: +81 98583 3527; Tel: +8198583 3527 

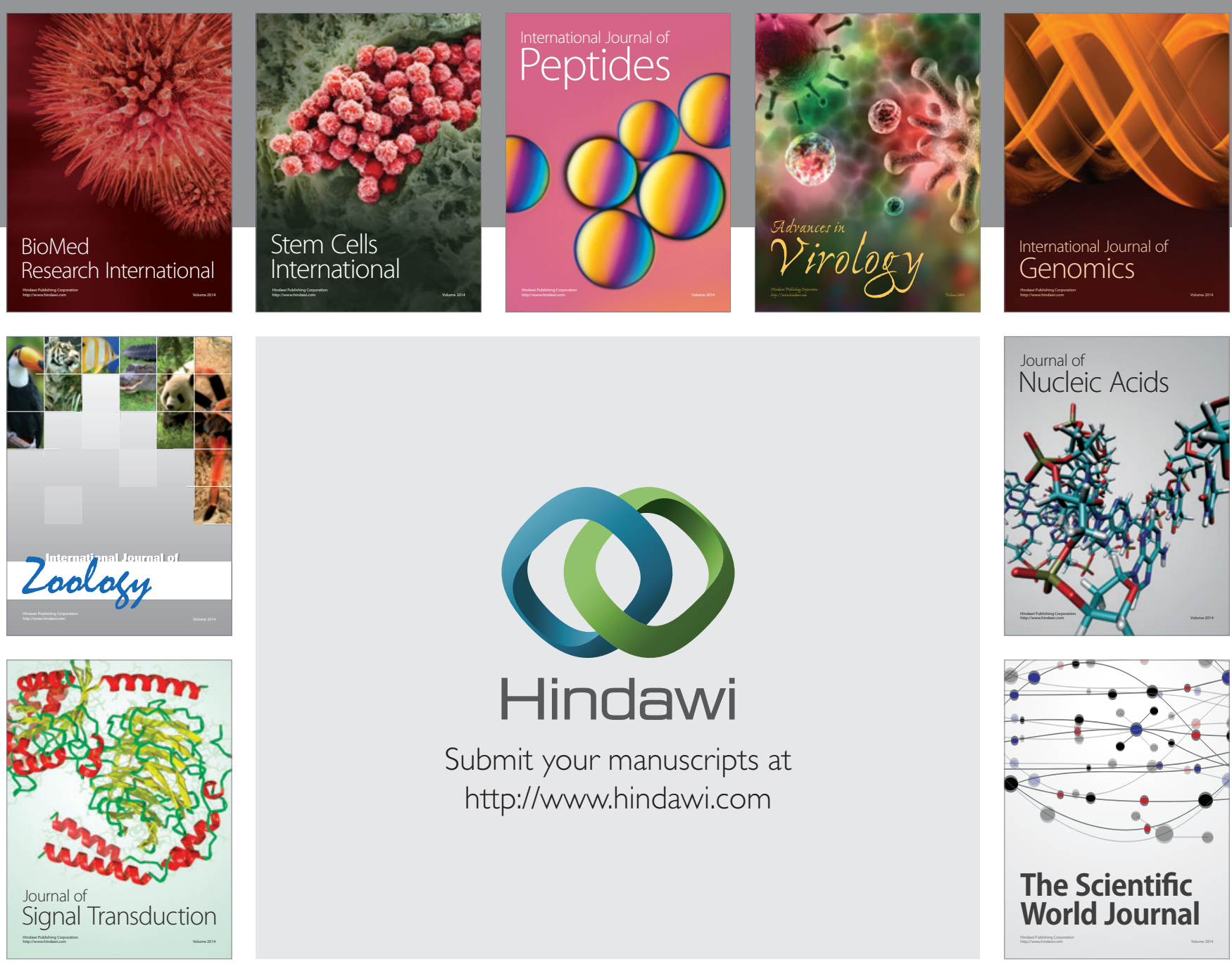

Submit your manuscripts at

http://www.hindawi.com
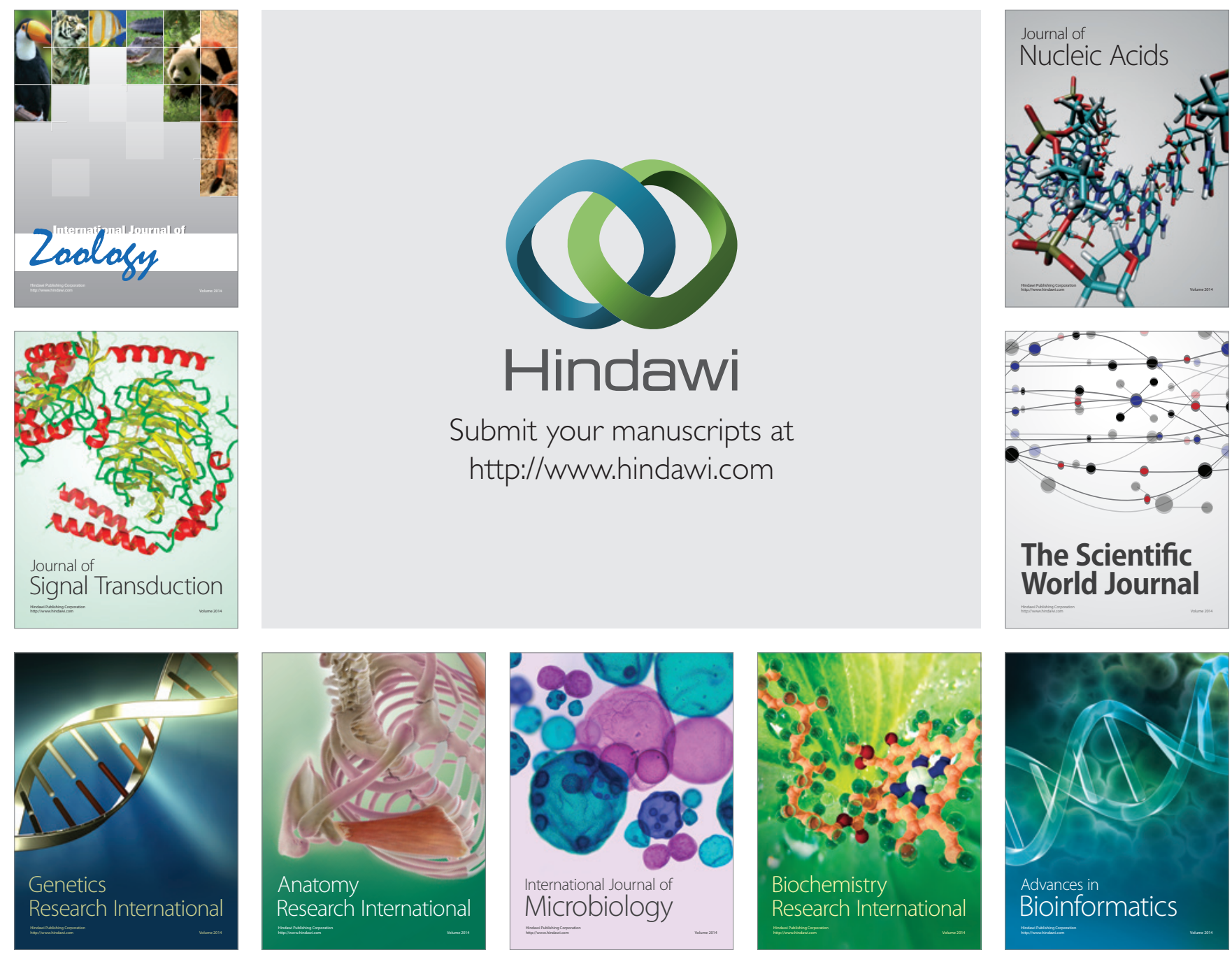

The Scientific World Journal
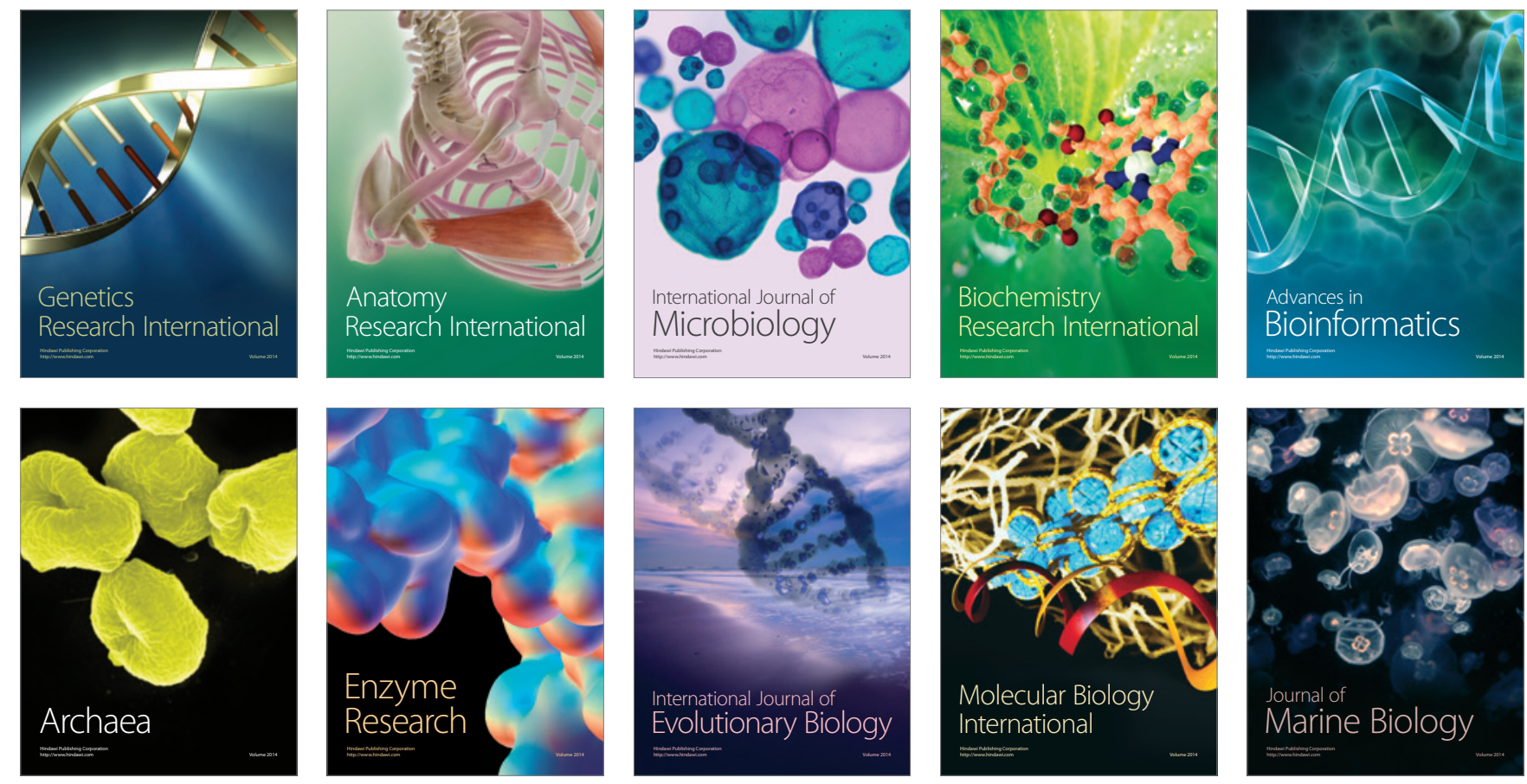